Recepción: 23 / 05 / 2018

Aceptación: 17 / 07 / 2018

(C) $(\Theta \Theta(-)$

Ciencias de la educación

Publicación: 01 / 08 / 2018

Artículo de investigación

\title{
Homeschooling una alternativa en educación
}

\section{Homeschooling an alternative in education}

\section{Homeschooling uma alternativa na educação}

\author{
Martha L. Avalos-Obregón ${ }^{\mathrm{I}}$ \\ luciaavalos@unach.edu.ec \\ María D. Avalos-Obregón II \\ mavalos@unach.edu.ec \\ Fabián Cazar del Pozo III \\ fbncazar29@gmail.com
}

Correspondencia: luciaavalos@unach.edu.ec

\footnotetext{
${ }^{I}$ Magister en Educación Parvularia, Licenciada en Ciencias de la Educación Profesora de Educación Básica, Docente Universidad Nacional de Chimborazo, Riobamba, Ecuador.

${ }^{\text {II }}$ Magister en Educación Parvularia, Licenciada en Ciencias de la Educación Profesora de Educación Básica Docente Universidad Nacional de Chimborazo, Riobamba, Ecuador.

III Magister en Gerencia de Proyectos Educativos y Sociales, Doctor en Ciencias Pedagógicas, Licenciado en Ciencias de la Educación, Profesor de Enseñanza Media en la Especialización De Psicología Educativa y Orientación, Docente del Instituto Tecnológico San Gabriel, Riobamba, Ecuador.
} 


\title{
Resumen
}

El homeschooling es la educación en casa dirigida por los padres, madres de familia o tutores para ejercer una práctica pedagógica en el hogar, con el fin de potenciar las habilidades y destrezas que poseen lo niños respetando su individualidad y ritmo de aprendizaje; el método empleado en esta investigación es descriptivo con un enfoque cualitativo que permitió recopilar relatos e historias de vida de 20 madres homeschooling participes del curso de pedagogía blanca en el período 2017-2018, considerando a la práctica de homeschooling como una alternativa educativa en diversos contextos, concluyendo las ventajas de esta práctica, por lo que los niños evidencian un control emocional, conciencia social, identidad y autonomía, desarrollo de la creatividad, apego por la naturaleza e interés para aprender otro idioma, el espacio para interpretar instrumentos musicales y demostrar sus habilidades deportivas tienen igual importancia que el desarrollo de habilidades numéricas o lógicas.

Palabras clave: educación familiar; educación comunitaria; educación extraescolar.

\begin{abstract}
Homeschooling is home education directed by parents, mothers or guardians to practice a pedagogical practice at home, in order to enhance the skills and abilities that children have respecting their individuality and pace of learning; the method used in this research is descriptive with a qualitative approach that allowed to compile stories and life stories of 20 homeschooling mothers participating in the white pedagogy course in the period 2017-2018, considering the practice of homeschooling as an educational alternative in various contexts, concluding the advantages of this practice, so that children demonstrate emotional control, social awareness, identity and autonomy, development of creativity, attachment to nature and interest to learn another language, space to interpret musical instruments and demonstrate their Sports skills are equally important as the development of numerical or logical skills.
\end{abstract}

Key words: family education; community education; extracurricular education. 


\title{
Resumo
}

O ensino domiciliar é uma educação domiciliar dirigida por pais, mães ou responsáveis para a prática de uma prática pedagógica em casa, a fim de aprimorar as habilidades e habilidades que as crianças possuem, respeitando sua individualidade e ritmo de aprendizagem; o método utilizado nesta pesquisa é descritivo com abordagem qualitativa que permitiu compilar histórias e histórias de vida de 20 mães de homeschooling participantes do curso de pedagogia branca no período 2017-2018, considerando a prática do homeschooling como alternativa educacional em diversos contextos, concluindo as vantagens dessa prática, para que as crianças demonstrem controle emocional, consciência social, identidade e autonomia, desenvolvimento da criatividade, apego à natureza e interesse em aprender outra língua, espaço para interpretar instrumentos musicais e demonstrar suas habilidades esportivas são igualmente importantes desenvolvimento de habilidades numéricas ou lógicas.

Palavras chave: educação familiar; educação comunitária; educação extracurricular.

\section{Introducción}

Homeschooling una alternativa en educación

Homeschooling una alternativa en educación en el curso de formación de pedagogía blanca promoción 8 en el período mayo 2016- mayo2017

\section{Historia del arte}

\begin{abstract}
Al hablar de temas educativos, de manera inherente se debe referir a la legislación que apoya este derecho a nivel mundial; siendo las Constituyente de cada país la carta garantista de dotar de derecho a la educación en un estatus históricamente reivindicado desde hace siglos, y se busca con ello satisfacer las necesidades y aspiraciones del individuo de formarse logrando un pleno desarrollo de su personalidad, especialmente respecto al menor de edad, quien es un individuo especialmente vulnerable que depende de un padre o tutor para cubrir sus necesidades y alcanzar un pleno desarrollo. Es, por tanto, un derecho fundamental que se encuentra reforzado a través de un deber de recibir la enseñanza, implementando el ordenamiento una serie de deberes para asegurar su ejercicio.
\end{abstract}


Siguiendo ese precedente, en el Reino Unido se promulgó la Education Act en 1996, que permite la educación en casa a cargo de los padres. No obstante, no la regula como una libertad total, sino que establece una serie de controles que permiten a las autoridades locales en materia de educación asegurarse de que los padres den cumplimiento al deber de educar a sus hijos en forma proporcionada con su edad, habilidad y aptitudes. Asimismo, dispone que las autoridaduedan exigir información sobre las medidas educativas que se han adoptado para el menor y, de concluir que la educación no es la adecuada, pueden ordenar la escolarización del niño. (Soberanes \& Trejo, 2011)

Rafael Navarro-Valls y Javier Martínez-Torrón consideran que, aunque pareciera que es la regla general, educación no es sinónimo de escolarización. Así mismo sostienen que el homeschooling es un fenómeno al alza que ha sido implementado en diversos países, entre ellos Estados Unidos, Canadá, Australia, Chile, Reino Unido, Irlanda, Austria, Hungría, Finlandia, Francia, Dinamarca y Bélgica. Sin embargo, cuando se permite la enseñanza en el hogar, no se trata de que el Estado se vea ajeno a lo que ocurre con los menores, sino lo contrario. A ese respecto, apuntan que los países que permiten el homeschooling tienen una regulación con diversos modelos de control público sobre la enseñanza que se proporciona por las familias, de tal modo que se garanticen los derechos de los padres sobre la educación de sus hijos, y a la par, el cumplimiento de los deberes estatales de enseñanza. (Navarro \& Martínez, 2011)

\section{En el Ecuador}

En relación con lo descrito anteriormente en referencia a la legislación que garantiza al acceso a la educación está contemplado en la Carta Magna en el Art 26.- La educación es un derecho de las personas a lo largo de su vida y un deber ineludible e inexcusable del Estado. Constituye un área prioritaria de la política pública y de la inversión estatal, garantía de la igualdad e inclusión social y condición indispensable para el buen vivir. Las personas, las familias y la sociedad tienen el derecho y la responsabilidad de participar en el proceso educativo

En concordancia con lo manifestado en el apartado anterior el Art 29.- El Estado garantizará la libertad de enseñanza, la libertad de cátedra en la educación superior, y el derecho de las personas de aprender en su propia lengua y ámbito cultural; legislación que apoya y garantiza la libertad 
que el padre de familia puede tener para ejercer pedagogía de manera responsable con sus hijos en el hogar siendo este uno de los posibles contextos de aprendizaje. (Asamblea Constituyente, 2008)

Estableciendo una comparación en las legislaciones expuestas se tiene claro que el derecho de la educación de un niño y adolescente es ineludible por parte de todos los actores que contribuyan su formación, sin que esto quiera decir que el estado o los progenitores afecten el bienestar de los niños.

El poder legal que un progenitor o representante tiene sobre su hijo, y de las decisiones que se puedan tomar para beneficio en su formación tienen que ser analizadas de una manera imparcial, sin apasionamiento, difícil porque se trata del presente y futuro de su hijo; para el estado es más fácil porque es una parte de un grupo muestra de una estadística determinada o de un caso aislado.

La práctica educativa que se realiza en casa, en la comunidad o en una institución tiene que conjugar un sinnúmero de elementos que respondan a la necesidad del contexto no a intereses particulares o peor a políticas públicas de momento.

\section{Homeschooling}

"Es imposible educar a los niños por mayor; la escuela no puede ser el sustitutivo de la educación individual" Alex Carrel. Premio nobel de medicina (1912)

Al hacer una consideración contextual se puede definir al homeschooling como la educación a tiempo completo de niños en y alrededor de la casa por parte de sus padres o custodios, o por tutores. (Bruce, 2010). Por lo tanto, la educación en casa, tiende a constituirse en la acción pedagógica previamente planificada y ejecutada por los progenitores o tutores que aplican un proceso de aprendizaje.

La educación en casa, no se trata de llevar la escuela a casa, es un proceso de relación empática entre los actores educativos, la casa se convierte en un constante laboratorio de ensayo, exploración y sobre todo de descubrimiento de habilidades. 
El proceso que demanda el ejercicio de homeshooling es sin lugar a duda es altamente innovador; el conocer el ritmo de aprendizaje de los hijos requiere más de una buena intención, se necesita interés por la acción educativa con la visión o subjetividad que cada hogar posee.

El hecho de ejercer una práctica educativa responsable en casa, es la esencia, la puesta en escena del cambio que se debe generar en la conceptualización de educación, no solo es la acción pedagógica de descifrar letras o saber fórmulas, es interpretar la realidad para transformarla, es convivir de manera armoniosa con nuestros iguales y con quienes consideramos diferentes también, eso no lo podemos hacer cuando hay exceso de niños en un determinado grado al cual se debe impartir grandes contenidos, no hay tiempo suficiente para una relación e interrelación libre entre los actores educativos.

Como todo proceso, el homeschooling también presenta cambios o modificaciones, es ahí donde nace unschooling, una actitud que refiere a la no escolarización, esta acción implica despojarnos de todo lo que hemos considerado como correcto en educación o por lo menos lo que nos ha permitido alcanzar ciertas metas cuantificables en la vida como un trabajo, un oficio u ocupación dentro del mercado laboral. Descolarizar nuestra mente compromete eliminar varios conceptos inherentes al accionar educativo, como contenidos, temas, evaluaciones, asignaturas y todo trasladar a que el niño solo aprenda mediante su propio descubrimiento, que su guía sea la creatividad, su horizonte la curiosidad, el arte su forma de expresión; pero quien comprende esta inusual forma de aprender, ¿estamos listos para enfrentar esta realidad?

\section{Homeschooling como alternativa en la educación}

Al respetar el ritmo y estilo de aprendizaje de cada uno de los hijos se está en la capacidad de responder a sus necesidades, sin obligar a realizar tareas que en una escuela se deben ejecutar en un tiempo determinado con un material proporcionado por la docente y con el rigor del cumplimiento de reglas y normas impuestas desde una autoridad que regenta el funcionamiento de la institución educativa.

En un ejercicio pleno y complaciente de lo que es educación se debe analizar con cuidado lo que realmente es el proceso de aprendizaje. 
Enseñar exige rigor metódico

Enseñar exige investigación

Enseñar exige respeto a los saberes de los educandos

Enseñar exige crítica

Enseñar exige estética y ética

Enseñar exige la corporificación de las palabras por el ejemplo

Enseñar exige riesgo, asunción de lo nuevo y el rechazo a cualquier tipo de discriminación

Enseñar exige crítica sobre la práctica

Enseñar exige el reconocimiento y la asunción de la identidad cultural (Freire, Pedagogía de la autonomía, 2004)

Al practicar un educación liberadora y autónoma, la escolarización no presentará problemáticas educativas; pero al no ejercer un aprendizaje propio construido desde la necesidad y perspectiva del niño; se pierde el horizonte y la naturaleza de una escuela; ante esta situación se presenta la educación en casa o homeshooling como una alternativa para la formación de niños y niñas

El proceso de aprender, en el que puede encender en el aprendiz una curiosidad creciente, que puede tórnalo más y más creador. Lo que quiero decir es lo siguiente: cuanto más críticamente se ejerza la capacidad de aprender tanto más se construye y desarrolla lo que vengo llamando "curiosidad epistemológica", sin la cual no alcanzaremos el conocimiento cabal del objeto. (Freire, Pedagogía de la autonomía, 2004)

Es alarmante que padres de familia o tutores se entusiasmen más al crear espacios y condiciones que fomenten esa curiosidad innata en el niño, el docente se dedica a cumplir planes curriculares de exigencia gubernamental, siempre atemorizada por una norma administrativa de sanción.

La institución educativa regentada por un Ministerio que responde a una política pública, independientemente si esta administración es consideraba buena, mala o pésima; la educación 
estará condicionada a esta calidad y estará lejos en convertirse en agente de transformación social; es esta sensación de insatisfacción lo que genera malestar en el núcleo familiar, y las conversaciones regulares incluyen desafiantes intercambios de ideas, propuestas que tienen los niños.

La educación vista desde los agentes que generan este ejercicio responsable sin lugar a duda deben ser parte de este proceso de construcción, sin embargo, se anula su criterio y se imparte contenido para que alcancen un destreza determinada o competencia que responde a su edad cronológica y al pensamiento de quien está a cargo de impartir clases.

\section{Razones para escoger homeschooling como alternativa en la educación}

La razón por la cual los padres eligen educar a sus hijos en casa todo o parte del tiempo son progenitores más comprometidos. Siendo esto así, podemos dividirlo en varias líneas generales. Hay insatisfacción con la disciplina o la seguridad en la escuela. Hay insatisfacción con la calidad del currículo ofrecido por la escuela. Hay objeciones religiosas o ideológicas a la experiencia de la educación como lo proveen los casos estatales. No es posible saber cuál es la razón principal. En muchos casos es una combinación de muchas razones que ha llevado a decisión para educar en casa. Pero la razón es la que se separa en la medida de lo posible y discuten en el orden dado (Bruce, 2010)

La responsabilidad que posee el progenitor es muy amplia, más aún cuando se tiene una visión de pertinencia en el transitar de la vida más allá de una instrucción, invita a una responsabilidad compartida en la formación del niño, cuando este equilibrio tiene una ruptura sea por la imagen que proyecta una institución educativa que procura mantener un régimen estricto, o con una connotación religiosa, o por ser indiferente a las inquietudes, problemas que tiene el niño, será cuestionada y como resultado se genera un resquebrajamiento elemental de convivencia entre los padres de familia y la institución; como alternativa se encuentra la educación en casa.

Determinar una causal específica resulta imposible, la variedad del desencanto del sistema educativo cubre varios aspectos; por lo que la solución ante esta desilusión se presenta la educación en casa. 
El bulliyng o acoso escolar figura también como una causa para que el padre en el cumplimiento pleno del rol protector eduque en casa a su niño víctima de esta problemática; bajo ningún concepto podemos pensar que el niño que sufre este acoso es el culpable por su debilidad ante las agresiones; pero lo que realmente es alarmante son las acciones que generan esta conducta ¿qué hace la escuela o comunidad ante este acontecimiento? ¿Qué hacen los padres para solucionar este problema? Para muchos la respuesta es la deserción y para otros homeschooling se presenta como la opción más acertada para combatir este problema

Una situación a un más alarmante e inimaginable es el abuso sexual. En el Ecuador es un mal que no parece tener fin, los progenitores no actúan por vergüenza o miedo a represalias, un caso en particular sucedió en la ciudad de Quito, y como un acto preparatorio a la honra de los niños se hizo colocar una placa en memoria de los niños del sexto C y llevará está frase: "Por las víctimas de abuso infantil en el sistema educativo". (El comercio, 2017). No solo reprochable, desgarrador como confiar a un sistema tan viciado la educación de nuestros niños. Por eso nuevamente nace la educación en casa como la mejor opción para garantizar el cumplimiento de los derechos fundamentales de los niños.

La normativa legal vigente en el Ecuador ampara la educación en casa, sin embargo, regula de tal manera que no se puede ejercer este derecho con libertad, se tiene que cumplir con la formalidad requerida, lo que limita a familias a la práctica de la educación en casa; acción que está amparada y desarrolla en otros países.

\section{Ventajas de homeschooling}

Mantener la identidad propia con una formación constructiva sin lugar a duda es parte del proceso de educación dice la identidad entendida como la relación contradictoria que somos nosotros mismos entre lo que heredados y lo que adquirimos, relación contradictoria en que a veces lo adquirimos en nuestras experiencias sociales, culturales, de clase, ideológica interfiere vigorosamente a través del poder de los intereses, emociones, de los deseos, de lo que llamamos "la fuerza del corazón", en la estructura hereditaria. (Freire, 2008). Al tener una educación en casa impartida por los progenitores o tutores se puede ir moldeando paulatinamente ese espíritu irreverente ante la injusticia, la inequidad social, pero sobre todo al ejercer plenamente el derecho 
a ser diferente, a convivir con una forma opuesta de generar educación, o por el simple hecho de buscar algo mejor.

El homesholling permite desarrollar al niño todas sus capacidades sin tener que rendir tributos a evaluaciones que no respetan proceso o estilos de aprendizaje propios, al masificar a los estudiantes el sistema pretende obediencia, silencio y complicidad de actos poco aceptables.

Los estudiantes que ejercen tutorías académicas en el hogar tienen mucho que ofrecer a universidades altamente selectivas en los Estados Unidos, una amplia gama de conocimientos y experiencias, confianza y facilidad en el trabajo con adultos, un sentido claro de sus fortalezas y debilidades académicas y un enfoque auto dirigido y decidido hacia el aprendizaje. (Bruce, 2010)

Experiencias como la de Paula Rothermel, investigadora británica que realizó su trabajo con más de 1000 familias homeshooler durante tres años, demostrando que los niños educados en casa manifestaron altos niveles de alfabetización y de habilidades sociales.

El empoderamiento de los jóvenes por su proceso de aprendizaje y el entusiasmo que mantienen por hacer cosas que le gustan, eventos de su agrado les asegura el éxito en diversos escenarios educativos, lo que sucede a la inversa con los jóvenes que asisten al sistema educativo tradicional pasan horas y horas para aprender contenidos que no son del contexto o de su interés, si no con la finalidad de aprobar un nivel o año; o en el mejor de los casos recibir una recompensa por parte de sus profesores

La práctica educativa centrada en el niño, es coherente porque le brinda que la importancia que tiene, le adjudica los créditos que solo su ingenio y creatividad pueden lograr; el niño nos enseña como aprende, nos enseña como construye su conocimiento que no está en un texto, por lo general está en la acción que realiza para entender o interpretar su realidad, no nuestra visión o concepto.

Al realizar homescholing el niño tiene la oportunidad de desplazarse con gran facilidad, su escenario de aprendizaje es el cuarto de baño, la cocina, la cochera, su jardín o el cuarto de lavado; tiene la oportunidad de interpretar videos, visitar museos, participar en eventos culturales, eventos que los niños que asisten a una escuela regular se están perdiendo. 


\section{Marco Metodológico}

\section{Método}

Descriptivo. - Consiste en describir el estado actual de casos hechos, fenómenos, personas o cosas, explicando sus distintas partes, cualidades, propiedades o circunstancias, explicando sus distintas partes, cualidades, propiedades o circunstancias, no sólo por sus atributos, sino más bien dando una idea completa del contexto, interpretando en forma real lo que se investiga (Arellano, 2000)

\section{Enfoque}

La investigación se basa en un paradigma cualitativo que asume el dinamismo de la realidad y de contextualización holística que busca la comprensión de los fenómenos sociales, en este caso en particular la práctica de homeshooling surge como una alternativa en la educación.

\section{Modalidad}

Campo. - En esta modalidad el investigador toma contacto en forma directa con la realidad, para obtener información de acuerdo con los objetivos del proyecto, para lo cual en este proyecto se contactó con las participantes del curso de pedagogía blanca (práctica de homeschooling) promoción número ocho, quienes, mediante video conferencias, relatos de vida que demuestran la práctica de homeschooling como una alternativa en la educación.

Documental. - Tiene el propósito detectar, ampliar y profundizar diferentes enfoques, teorías, conceptualizaciones y criterios de diversos autores sobre una cuestión determinada. (Herrera \& Medina, 2004)

\section{Técnica e instrumentos para la recolección de datos}

La técnica que se aplicó historias de vida, relatos mediante video conferencias que permiten demostrar las diferentes prácticas de homeschooling. 


\section{Población}

Madres del curso de pedagogía blanca (practica de homeschooling) promoción 8 en un total de 20 participantes

Procesamiento para el análisis e interpretación de resultados

El proceso a seguir será el siguiente:

Obtención de datos

Procesamiento de datos

Análisis de los resultados

Conclusiones y Recomendaciones

\section{Análisis de los relatos de vida de las madres homeschooler}

Para María, el homeshooling es una herramienta educativa válida que podría estar regulada por el Estado democrático actual, para mejorar la oferta y la calidad de la enseñanza y reducir así el absentismo o el abandono escolar.

"Ante todo, me gustaría resaltar que no se trata de un enfrentamiento entre la escuela y homeschooling, entre docentes y padres, sino que se intenta acercar las dos realidades para el sistema educativo actual pueda acoger todas las posibles formas de enseñanza. Deseo que cada uno de mis hijos, que son cinco: Inés, Lucia, André, Emilo y Dagne; puedan tener la educación que se merece, bien sea en la escuela o fuera, teniendo presente que lo primordial es la futura generación. Actividades como realizar una conserva de tomate, diseño y costura de una falda, la construcción de un barco de vela o un coche propulsado por aire, se convierte en toda una aventura, la iniciativa, la creatividad se pone a prueba pero sobre todo son felices, no compiten por sobresalir, al contrario comparten para hacer un trabajo en conjunto, al ser maestra primaria, doctora en educación, es lo que me hace discernir con claridad que la práctica del homeschooling tiene muchas ventajas en relación a la educación impartida en la escuela regular. Yo practico el homeschooling desde hace 10 años; las ventajas son múltiples, lo que hace falta es apoyo del 
Estado para que esta práctica pueda ser reconocida y remunerada.". (María 2017, cuaderno de notas)

El homeschooling es considerado como un enfoque innovador de la educación, visto como algo placentero y divertido, lo que es contrario a la situación actual de las aulas comunes de una escuela regular; el estudiante en la práctica del homeschooling aprende de manera flexible, natural, de manera espontánea e inmerso en su propio medio social, cultural y tecnológico, siguiendo sus intereses, sus talentos y sus aptitudes innatas.

En una institución educativa se habla tanto de la construcción del propio conocimiento a través de aprendizaje basado en problemas, o del tan anhelado aprendizaje significativo; en ese momento existe una brecha entre la planificación y práctica al ejecutar el tema; de manera teórica hasta cierto punto utópica el docente pretende hacer la clase activa, participativa para consolidar los objetivos que se planteó; pero cuan significativo es mostrar en lámina o vídeo las frutas cuando más significativo resulta realizar una coctel o ensalada de frutas, de esta manera el tema de clase se construiría de una manera más relajada; sin embargo la cantidad de niños y lo que esta actividad demanda no se ejecuta; pero lo que en clase resulta imposible en la práctica homeschooling es un ejercicio común.

Richard Gerver, tras realizar un viaje a Disneyland®, quedó sorpendido por cómo los niños podían esperar por horas para ingresar a un juego que solo les duraba 3 minutos; con esta premisa este profesor británico de gran reconocimiento mundial en el ámbito educativo, ha creado una escuela que desafía a todo currículo actual; esta escuela; es toda una ciudad, dirigida por un alcalde; tiendas, restaurantes, emisora de televisión, radio, entre otras dependencias; todas estas administradas por niños. Cuantas veces en la escuela regular permitimos al niño decidir simplemente entre los colores para pintar un dibujo; inimaginable pensar que un niño organice la colación o almuerzo de la semana que se reparte en el colegio. Ante esto Gerver propone una educación flexible y personalizada; lo lamentable es que no se puede hablar de una educación personalizada en un grupo de 20 hasta 45 estudiantes por salón de clase.

Para Azucena la práctica de homeschooling se ha convertido en su forma de vida, en la consolidación integral como madre y mujer. 
"Soy una madre homeschooler más de doce años; mis dos hijos dejaron de ir al cole cuando en el rostro de ellos vi que el esfuerzo que ponían para realizar una tarea escolar que era enviada a casa se convirtió en sufrimiento; no podía permitir que mis hijos sean valorados por una calificación que no reflejaba su creatividad, su poder de elección, peor aun cuando la nota tenía el sello inconfundible de un docente muy autoritario. Conversamos con mi esposo y decidimos sacarlos del cole y ya está, así fue. Desde ese día en casa empezamos a disfrutar del acompañamiento integral de nuestros hijos; hubo días buenos, otros fueron fatales; pero en el homescooling encontré muchas ventajas, mis hijos son más sociables, reflexionan antes de hacer las cosas o cuando tiene un problema lo resuelven super rápido, tienen un apego por el cuidado por la naturaleza y respeto por sus iguales; dominan varios idiomas, también les gusta practicar varios instrumentos musicales. En el homeschooling solo veo ventajas, a mi familia le cambio la vida para bien." (Azucena 2017, cuaderno de nota)

Para Mireia entre los principales objetivos curriculares del homesholling en concordancia con el trabajo tutorial de sus padres es la búsqueda de desarrollo completo del niño, su plan personal de vida. Es difícil concretar una lista común entre las familias homeschooler, pero por lo general se trata de alcanzar las siguientes características:

“ Yo busco en mis hijos y ahora nietos porque soy una madre y abuela homeschooling, lo bueno es que se trabaja de manera transversal las habilidades de los chicos; investigan la propia realidad, día a día; se fomentan las inteligencias múltiples, la de lengua y mate son tan importantes como la ecológica o musical; se respeta el ritmo del chico, promover un aprendizaje significativo, se realizan proyecto en función a su interés; se desarrolla en un entorno relajado; se integra el aprendizaje dentro de la comunidad o barrio con excursiones, investigaciones o salidas culturales. Mira es fácil si quieres niños felices, creativos, reflexivos, independiente, soñadores practica homeschooling; sin quieres niños sin creatividad como dice Robison que estudien en una escuela regular.” (Mireia 2017, cuaderno de nota)

El trabajo integral con el niño permite que se desarrolle en todos sus ámbitos, que evidencie sus habilidades sin temor a ser criticado, se fomenta la significatividad de las acciones, pero sobre todo del sentimiento del niño, lo que permite conectar con lo más íntimo del ser humano le ayuda 
a conocer, a admirar lo bello y esta acción es liberadora, enseñándonos el complicado arte de vivir.

Los relatos de historias de vida reflejan una condición preocupante del sistema educativo de varios países teniendo como constante esta la acción atentatoria contra la creatividad; además el irrespeto el estilo de aprender de cada niño, existiendo también información sobre la práctica de una educación de tipo conductista, que como estrategia emplea el castigo; por todo lo descrito anteriormente surge homeschooling como una alternativa en la educación; es preocupante que padres de familia o representantes legales tomen la tutela para que sus hijos aprendan; que se involucren en descubrir elementos activos que potencien las habilidades de sus hijos sin coartar su deseo de descubrimiento o creatividad; con un plus adicional de autonomía y libertad.

Los relatos reflejan que tomaron la alternativa del homeschooling porque la escuela mata la creatividad lo que no permite que el niño manifieste su verdadero interés; la escuela regular masifica el pensamiento mediante estructuras pre- establecidas como un horario rígido, evaluaciones que solo consideran el ámbito cognitivo, procesos educativos que fomentan el silencio y una extrema disciplina que es asociada con la quietud y buen comportamiento; también se refleja un irrespeto por el pensamiento del niño o su constante movimiento por descubrir.

Sin lugar a duda un elemento que a todo profesor nos debe llamar a la reflexión es el deseo de madres y representantes legales que sin tener formación docente conocen de elementos pedagógicos, proyectos educativos, componentes psicológicos, estrategias metodológicas para aproximar o guiar a sus tutelados o hijos en el camino del aprendizaje. Sobre todo generan un aprendizaje respetuoso que potencian habilidades no solo cognitivas, sino destrezas sociales, adquisición de varias lenguas, interpretación de instrumentos musicales, conciencia ecológica y despiertan un gran sentido por el interés cultural, por la práctica de la paz aportando a la construcción de una sociedad más justa, elementos que se trata muy poco en las escuelas.

Homeschooling nace por la necesidad que brindar al niño un ambiente óptimo para el proceso de aprendizaje no con fin último sino como hilo conductor para descubrir y redescubrir nuevos elementos que posibiliten un enriquecimiento cultural atípico que no responde a estándares únicos se ve desde la diversidad que responde a la integralidad del niño; no se fracciona a un 
aprendizaje disciplinar o por áreas; se integra todo saber en un objetivo que es lo que niño necesita en ese momento.

\section{Conclusiones}

El homeschooling debe ser considerado por la normativa vigente de todo estado que se desarrolle en democracia como una alternativa en la educación por las ventajas que presenta su aplicación; el integrar contenidos reales que respondan a su contexto, el respeto del ritmo de aprendizaje del niño; considerar el interés que posee para aprender determinado contenido; brindar apoyo de igual manera si desea interpretar un instrumento musical o resolver una fórmula química; la capacidad que tiene de desenvolverse en un escenario cultural; las ventajas de la práctica del homeschooling varía entre familia y familia; sin embargo tiene un común denominador que es la gran habilidad social que poseen estos niños en relación a los niños que asisten a clases regulares.

Las familias que han optado por la práctica del homeschooling, refieren a que la escuela donde sus hijos estaban respondían a un currículo totalmente desactualizado; el sistema educativo no mostraba el mínimo respeto por el verdadero interés del niño o peor aún motivar la creatividad que posee el niño de manera innata; muchos padres de chicos que sufrieron bullying o acosos escolar quienes lo único que desean es abandonar sus estudios, encontraron en el homeschooling una solución acertada para enfrentar este problema. Es preocupante saber que la escuela, lugar donde se debería educar, no está cumpliendo este objetivo

El Homeschooling no está contrapuesto, no es un ataque a la escuela. Al contrario es la educación en familia, que lo busca es su reconocimiento, la práctica de este ejercicio pedagógico permitirá mejorar la relación entre una institución educativa, la familia y la comunidad; el ejercicio pleno de los derechos en todo estado democrático debe ser garantista de esta alternativa en la educación que se presenta como solución ante el absentismo o abandono escolar; el sistema actual educativo debe innovarse; estructurar cambio verdaderos, reales que cumplan más allá de lo académico, porque educarse no solo es instruirse o llenar su memoria de contenidos; educarse es poner en manifiesto lo mejor de cada ser humano. 


\section{Referencias Bibliográficas}

Arellano, Enrique. (2000). Investigación Científica. Loja: Ediciones COSMOS.

Asamblea Constituyente. (2008). Constitución del Ecuador. [documento en línea]. disponible de: www.wipo.int/edocs/lexdocs/laws/es/ec/ec030es.pdf [consultada: 2017, noviembre,2]

Bruce, C. (2010). Home Schooling in full view. Connecticut: IAP Information Age Publishing. El comercio. (19 de 06 de 2017). ElComercio.com. Obtenido de ElComercio.com. [documento en línea]: disponible en: http://www.elcomercio.com/actualidad/colegio-quito-placa-abusosexualninos.html [consultada. 2017, noviembre, 3]

Freire, Paulo. (2004). Pedagogía de la autonomía. Sao Paulo: Ediciones: Paz y Tierra.

Freire, Paulo. (2008). Cartas a quien pretende enseñar. Argentina: Ediciones: Artes gráficas del Sur.

Herrera, Luis., \& Arnaldo, Medina. (2004). Tutoría de la Investigación Científica. Ambato: Ediciones Corona.

Navarro-Valls, Rafael., \& Martínez, Javier. (2011). Conflictos entre conciencia y ley. Las objeciones de conciencia. Madrid: Iustel.

Soberanes, José María., \& Trejo, Luis Alberto. (2011). Educación escolarizada vs. educación en casa: Revista Mexicana de Derecho Constitucional. No25. pp354-365.

\section{Anexos}

Martha Lucia Avalos Obregón, licenciada en ciencias de la Educación. Profesora en Educación Básica. Magister en Educación Parvularia. Docente Titular de la Carrera de Educación Inicial de la Facultad de Ciencias de la Educación Humanas y Tecnologías de la UNACH. Doctorante del Programa en Innovación Didáctica y Formación del Profesorado de la Universidad de Jaen España.

Calle 10 de agosto y Morona esquina. 0995259902 luciaavalos@unach.edu.ec

María Dolores Avalos Obregón, licenciada en Ingles. Master en Docencia Universitaria y Administración Educativa. Master en Estudios Ingleses Avanzados Lenguas y Culturas en Contacto. Doctorante del Programa Interuniversitario en Lenguas y Culturas. España. Docente Titular de la Carrera de Pedagogía de los Idiomas Nacionales y Extranjeros. 
Calle Larrea 26-40 entre argentinos y Junín. 0987590728 mavalos@unach.edu.ec

Fabián Ramiro Cazar del Pozo licenciado en Psicología Educativa, Diplomado en Liderazgo y

Gerencia, Master en Gerencia de Proyectos Educativos y Sociales, Doctor en Ciencias Pedagógicas. Cuba. Vicerrector Académico del ITS “San Gabriel”.

Calle Loja y Chile.0983811385 fbncazar29@gmail.com 